\title{
DIFFUSION LIMIT OF THE VLASOV-POISSON-FOKKER-PLANCK SYSTEM*
}

\author{
NAJOUA EL GHANI ${ }^{\dagger}$ AND NADER MASMOUDI ${ }^{\ddagger}$ \\ Dedicated to the sixtieth birthday of Professor Andrew Majda
}

\begin{abstract}
We study the diffusion limit of the Vlasov-Poisson-Fokker-Planck System. Here, we generalize the local in time results and the two dimensional results of Poupaud-Soler [F. Poupaud and J. Soler, Math. Models Methods Appl. Sci., 10(7), 1027-1045, 2000] and Goudon [T. Goudon, Math. Models Methods Appl. Sci., 15(5), 737-752, 2005] to the case of several space dimensions. Renormalization techniques, the method of moments and a velocity averaging lemma are used to prove the convergence of free energy solutions (renormalized solutions) to the Vlasov-Poisson-FokkerPlanck system towards a global weak solution of the Drift-Diffusion-Poisson model.
\end{abstract}

Key words. Hydrodynamic limit, Vlasov-Poisson-Fokker-Planck system, Drift-Diffusion-Poisson model, moment method, velocity averaging lemma, renormalized solutions.

AMS subject classifications. 35Q99, 35B25, 45K05.

\section{Introduction}

In this paper, we study the diffusion limit of the Vlasov-Poisson-Fokker-Planck system (VPFP for short). This generalizes the study done in Poupaud-Soler [30] and in Goudon [17] where the same problem was treated in the two-dimensional case. In these works the authors proved the convergence of regular enough solutions to the VPFP system towards a solution to the Drift-Diffusion-Poisson model, which is a standard model for plasmas and very well suited for numerical computations. In [30] Poupaud and Soler established convergence on a small enough time interval in 2 and 3 dimensions, under a suitable regularity assumption on the initial data. Here, we wish to prove a global convergence result, without any restriction on the time interval or on the dimension.

We consider the following rescaled VPFP system on $\mathbb{R}^{d} \times \mathbb{R}^{d}$ where $d \geq 2$,

$$
\left\{\begin{array}{l}
\partial_{t} f^{\varepsilon}+\frac{1}{\varepsilon} v \cdot \nabla_{x} f^{\varepsilon}-\frac{1}{\varepsilon} \nabla_{x} \phi^{\varepsilon} \cdot \nabla_{v} f^{\varepsilon}=\frac{\mathcal{L}_{F P}\left(f^{\varepsilon}\right)}{\varepsilon^{2}} \\
\mathcal{L}_{F P}(f)=\nabla_{v} \cdot\left(\beta v f+\sigma \nabla_{v} f\right) \\
-\triangle \phi^{\varepsilon}=\rho^{\varepsilon}-p(x)
\end{array}\right.
$$

where $\varepsilon$ is a small parameter related to the mean free path, $f^{\varepsilon}(t, x, v) \geq 0$ denotes the scalar distribution of particles, $\rho_{\epsilon}=\int_{\mathbb{R}^{d}} f_{\epsilon} d v$ is the density of electrons, and $p(x)$ is the density of a background of positive charges which is assumed to be fixed. Here $\sigma>0$ is the thermal diffusion coefficient and $\beta>0$ is the friction coefficient. For simplicity, we take $\sigma=\beta=1$. The time variable $t$ is nonnegative, the position $x$ belongs to $\mathbb{R}^{d}$ and the velocity $v$ belongs to $\mathbb{R}^{d}$ (see $[9]$ for a derivation of the model and its scaling).

The VPFP is one of the fundamental systems used in plasma physics. It models the evolution of the distribution function $f(t, x, v)$ of electrons which are subject to the electrostatic force coming from their Coulomb interaction and to a Brownian

\footnotetext{
*Received: February 1, 2009; accepted (in revised version): April 3, 2009.

†Département de Mathématiques, Faculté des Sciences de Tunis, TN-2092 Tunis, Tunisia (Najoua.ElGhani@fst.rnu.tn).

${ }_{\ddagger}^{\ddagger}$ Courant Institute, New York University, 251 Mercer St, New York NY 10012, USA (masmoudi@cims.nyu.edu).
} 
force which models their collisions. The electrostatic force is responsible for the selfconsistent force term $\nabla_{x} \phi^{\varepsilon} \cdot \nabla_{v} f^{\varepsilon}$ where $\phi^{\varepsilon}$ solves the Poisson equation. The Brownian force is modeled by the Fokker-Planck term $\mathcal{L}_{F P}\left(f^{\varepsilon}\right)$. We point out that there are other ways of modeling the collisions, for instance by a Boltzmann kernel (see [24] for some related results about the diffusion limit.)

We also point out that there is an attractive VPFP which is also widely used in stellar physics. For that case the repulsive electrostatic force is replaced by the attractive gravitational force, which is responsible for a change of sign in the Poisson equation. In this paper, we only consider the repulsive case.

There are many works dealing with the existence and uniqueness of solutions to the VPFP system as well as to systems close to it such as the Vlasov-Poisson system (VP). As we are only interested in VPFP, we will not detail results about VP. Global existence results for VPFP were obtained by Neunzert, Pulvirenti and Triolo [25] in $2 D$ using some probabilistic methods. This result was also obtained by Degond [11] using deterministic arguments, when $\beta=0$. Degond also studied the convergence of solutions to VPFP towards a solution to VP when the diffusion $\sigma$ goes to zero. Classical solutions were also constructed by Victory and O'Dwyer [36] in 2D and by Rein and Weckler [33] globally for small data (or for nearly neutral data) in 3D. Existence and uniqueness of global smooth solutions to VPFP in 3D is due to Bouchut [5]. These solutions are such that the initial data $f_{0}$ satisfies $f_{0} \in L^{1} \cap L^{\infty}\left(\mathbb{R}^{6}\right)$ and $|v|^{m} f_{0} \in L^{1}\left(\mathbb{R}^{6}\right)$ and the solution $(f, E=\nabla \phi)$ satisfies that $f \in C\left([0, T) ; L^{1}\left(\mathbb{R}^{6}\right)\right)$ and $E \in L^{\infty}\left((0, T) \times \mathbb{R}^{6}\right)$ for all $T>0$. The proof was inspired by the result of Lions and Perthame concerning VP [22] where global existence and uniqueness of solution were proved for VP (see also Pfaffelmoser [28] for a different proof and the simplifications in Schaeffer [34] and Horst [19]). Also, in [27], optimal time decay of the solution was obtained.

Parallel to the results about classical solutions, there are many papers dealing with weak solutions. We can mention for instance DiPerna-Lions [12, 13] where similar systems were considered, namely Vlasov-Poisson type systems and Boltzmann-FokkerPlanck. The two main ideas of DiPerna-Lions are the use of the averaging lemma to gain some compactness on $v$ averages of $f$ and the use of renormalization techniques to give sense to terms which are otherwise not well defined in the distribution sense. Let us also mention the work of Victory [35], Carrillo and Soler [8], and Bouchut [6] where some smoothing effect was also observed. There are also results about the global existence of solutions with measures as initial data in 1D due to Zheng and Majda [37] (see also [23]). These solutions have some similarity with vortex sheet initial data for 2D Euler.

In this paper, we will deal with renormalized solutions as constructed by DiPerna and Lions [12], which we also call free energy solutions. The terminology was for instance used by Bouchut and Dolbeault $[15,7]$. We insist on the fact that we only assume that the initial data has a finite free energy (see the definition below). It is important to insist on the fact that for more regular initial data (or more precisely for initial data such that $f_{0}$ is also in $L^{\infty}$ ), one can define the solution in the weak sense without the need of renormalizing it since the product $f E$ fully makes sense. In that case, the solutions we are considering are even unique if for instance $f_{0} \in L^{1} \cap L^{\infty}\left(\mathbb{R}^{6}\right)$ and $|v|^{m} f_{0} \in L^{1}\left(\mathbb{R}^{6}\right)$. However, even in this case one has to use renormalization techniques to pass to the limit since we can only use bounds which are uniform in $\epsilon$ to pass to the limit, and it seems to us that the free energy is the only quantity which is uniformly bounded. Notice that due to the friction term, one cannot use the maxi- 
mum principle to deduce that the $L^{\infty}$ norm of $f$ is uniformly bounded. It seems to us a nice open question to see whether one can obtain bounds for $f$ in $L^{\infty}$, for instance, which are uniform in $\epsilon$ using the representation formula given by Bouchut [5, 6]. Another way of approaching the problem is to use the result of Pulvirenti and Simeoni [31], which uses a stochastic differential extension of the notion of characteristics used in the work of Pfaffelmoser [28]. It gives an $L^{\infty}$ estimate on the density $\rho=\int f d v$ which is uniform in $\sigma$ and $\beta$, if they are smaller than some $\sigma_{0}$ and $\beta_{0}$ and the data is compactly supported in $v$. Here, we are actually in the case where both $\sigma$ and $\beta$ go to infinity. In any case, we did not pursue this direction since it was not necessary for our problem, and the notion of free energy (or renormalized) solutions seems very natural for this problem.

Concerning the asymptotic behavior of the solutions, we mention for instance [7, $9,3]$ where the long time behavior is studied. Here we are interested in hydrodynamic limits. There are two very important scalings of the VPFP (see [30]). The first one is called the low field limit (or the drift-diffusion limit, or the parabolic limit). This is actually the case we consider in this paper. There are many results about this limit. We refer to Poupaud [29] for the case of semi-conductor Boltzmann, and to Poupaud and Soler [30] and Goudon [17] for the case of VPFP. In these two works only the $2 \mathrm{D}$ case was considered (or the $3 \mathrm{D}$ case locally in time). One of the difficulties to extend their result to higher dimensions was that the term $f E$ can not be uniformly bounded. Here, the main idea to extend the result to higher dimensions is the use of renormalization techniques and averaging lemma in addition to estimates coming from the entropy dissipation. There are also many works dealing with the second scaling, namely the high field limit (or the drift limit, or the hyperbolic limit) [26, 1, 4, 18]. The high field limit leads to a hyperbolic system.

\section{Main result}

2.1. Free energy. As discussed in the introduction, we will work with solutions to the VPFP system which are defined in the renormalized sense. Before stating our main result about the VPFP system, let us mention that Drift-Diffusion-Poisson model can also be derived from other singular limits. We refer for instance to [24] where the Drift-Diffusion-Poisson model is derived from a semiconductor BoltzmannPoisson system and where the notion of free energy solutions was also used.

We supplement the VPFP system (1.1) with an initial data which is assumed to be known and depend on the mean free path $\varepsilon$ :

$$
f^{\varepsilon}(t=0, x, v)=f_{0}^{\varepsilon}(x, v) .
$$

Remarking that the Fokker-Planck operator can be rewritten as follows

$$
\mathcal{L}_{F P}(f)=\nabla_{v} \cdot\left(e^{-\frac{|v|^{2}}{2}} \nabla_{v}\left(f e^{\frac{|v|^{2}}{2}}\right)\right),
$$

we can guess on formal grounds that the penalization $\varepsilon \rightarrow 0$ leads to

$$
f^{\varepsilon}(t, x, v) \simeq \rho(t, x) M(v),
$$

where $M$ is the normalized Maxwellian with zero mean velocity:

$$
M(v)=\frac{e^{-\frac{|v|^{2}}{2}}}{(2 \pi)^{\frac{d}{2}}} .
$$



$f^{\varepsilon}$

Let us also define the charge and current densities associated to the distribution

$$
\rho^{\varepsilon}(t, x)=\int_{\mathbb{R}^{d}} f^{\varepsilon}(t, x, v) d v, J^{\varepsilon}(t, x)=\frac{1}{\varepsilon} \int_{\mathbb{R}^{d}} v f^{\varepsilon}(t, x, v) d v .
$$

In the case where we take the background of positive charges $p(x) \equiv 0$, the free energy functional is defined by

$$
\mathcal{E}^{\varepsilon}(t)=\int_{\mathbb{R}^{d}} \int_{\mathbb{R}^{d}} f^{\varepsilon}\left(\frac{|v|^{2}}{2}+\frac{\phi^{\epsilon}}{2}+\log \left(f^{\varepsilon}\right)\right) d v d x
$$

We recall here that $\phi^{\epsilon}$ is given by

$$
\phi^{\epsilon}=\Phi * \rho^{\epsilon}=\int \Phi(x-y) \rho^{\epsilon}(y) d y,
$$

where $\Phi$ is the fundamental solution of the Laplace equation, namely $-\Delta \phi=\delta_{0}$. It is given by

$$
\Phi(x)=\left\{\begin{array}{lr}
-\frac{1}{2 \pi} \log |x| & d=2 \\
\frac{1}{d(d-2) \omega_{d}} \frac{1}{|x|^{d-2}} & d \geq 3
\end{array}\right.
$$

where $\omega_{d}$ denotes the volume of the unit ball in $\mathbb{R}^{d}$. In particular in the case where $d=3, \Phi(x)=\frac{1}{4 \pi} \frac{1}{|x|}$.

Notice that one can also write

$$
\int_{\mathbb{R}^{d}} \int_{\mathbb{R}^{d}} f^{\varepsilon} \frac{\phi^{\epsilon}}{2}=\frac{1}{2} \int_{\mathbb{R}^{d}} \rho^{\epsilon} \phi^{\epsilon}=-\frac{1}{2} \int_{\mathbb{R}^{d}} \phi^{\epsilon} \Delta \phi^{\epsilon} .
$$

We are then tempted to integrate by parts and write it as $\frac{1}{2} \int_{\mathbb{R}^{d}}\left|\nabla \phi^{\epsilon}\right|^{2}$. This is actually justified if $d \geq 3$. However, when $d=2$ this is in general wrong since $\nabla \phi^{\epsilon}$ is only in $L^{2, \infty}$ due to the fact that $\nabla \Phi=-\frac{1}{2 \pi} \frac{x}{|x|^{2}}$ and hence only decays like $\frac{1}{|x|}$ when $d=2$.

When $p(x) \equiv 0$ in the system (1.1), only the self consistent interaction of the electrons with themselves is taken into account. If one takes also into account their interaction with a fixed background of positive charges then the Poisson equation $-\Delta \phi^{\epsilon}=\rho^{\epsilon}$ has to be replaced by $-\Delta \phi^{\epsilon}=\rho^{\epsilon}-p(x)$. If we also make the global neutrality assumption, namely

$$
\int_{\mathbb{R}^{d}} p(x)=\int_{\mathbb{R}^{d}} \int_{\mathbb{R}^{d}} f(x, v),
$$

then the potential energy term can be written as

$$
\frac{1}{2} \int_{\mathbb{R}^{d}}\left(\rho^{\epsilon}-p(x)\right) \phi^{\epsilon}=-\frac{1}{2} \int_{\mathbb{R}^{d}} \Delta \phi^{\epsilon} \phi^{\epsilon}=\frac{1}{2} \int_{\mathbb{R}^{d}}\left|\nabla \phi^{\epsilon}\right|^{2}
$$

and this now holds in any dimension $d \geq 2$ since now $\nabla \phi^{\epsilon}$ has a better decay when $x$ goes to infinity.

In the following we will always make the global neutrality assumption (2.7) and hence the free energy will be given by 


$$
\mathcal{E}^{\varepsilon}(t)=\frac{1}{2}\left\|\nabla_{x} \phi^{\varepsilon}\right\|_{L^{2}\left(\mathbb{R}^{d}\right)}^{2}+\int_{\mathbb{R}^{d}} \int_{\mathbb{R}^{d}} f^{\varepsilon}\left(\frac{|v|^{2}}{2}+\log \left(f^{\varepsilon}\right)\right) d v d x .
$$

From mathematical point of view, this slightly simplifies the proof of a priori estimates in dimension $d=2$ since we do not have to bound the potential energy from below. In the case $p=0$ and $d=2$, we refer to [17] where a bound from below on the potential energy is given using the fact that $|x| \rho^{\epsilon}$ is also controlled in $L^{1}$.

\subsection{Statement of the main result}

We will assume that the sequence of initial data satisfies:

A1: $\quad f_{0}^{\varepsilon} \geq 0, \quad \int_{\mathbb{R}^{d}} \int_{\mathbb{R}^{d}} f_{0}^{\varepsilon}\left(1+|x|+\frac{|v|^{2}}{2}+\left|\log \left(f_{0}^{\varepsilon}\right)\right|\right) d v d x<C$,

for some constant $C>0$ independent of $\varepsilon$.

A2: $\quad \int_{\mathbb{R}^{d}} \int_{\mathbb{R}^{d}} f_{0}^{\varepsilon} d v d x=\int_{\mathbb{R}^{d}} p(x) d x$ and $\nabla_{x} \phi_{0}^{\varepsilon}$ is uniformly bounded in $L^{2}\left(\mathbb{R}^{d}\right)$ where $\phi_{0}^{\varepsilon}=\Phi *\left(\rho_{0}^{\varepsilon}-p\right)$.

The main result of the paper is the following

Theorem 2.1. Assume that assumptions $\boldsymbol{A} 1$ and $\boldsymbol{A} 2$ are satisfied. Let $\left(f^{\varepsilon}, \phi^{\varepsilon}\right)$ be a free energy, renormalized solution (see the definition below) of the VPFP system (1.1)-(2.1). Then,

$$
\begin{aligned}
f^{\varepsilon} \rightarrow \rho M(v) & \in L^{1}\left(0, T ; L^{1}\left(\mathbb{R}^{d} \times \mathbb{R}^{d}\right)\right), \\
\phi^{\varepsilon} \rightarrow \phi & \in L^{2}\left(0, T ; W_{l o c}^{1, p}\left(\mathbb{R}^{d}\right)\right) ; \quad \forall p<2 .
\end{aligned}
$$

In particular, $\rho^{\varepsilon}$ converges weakly in $L^{1}\left(0, T ; L^{1}\left(\mathbb{R}^{d}\right)\right)$ towards $\rho$ and $(\rho, \phi)$ is a weak solution of the Drift-Diffusion-Poisson system

$$
\left\{\begin{array}{l}
\partial_{t} \rho+\nabla_{x} \cdot J=0 \\
J=-\nabla_{x} \rho-\rho \nabla_{x} \phi \\
-\triangle_{x} \phi=\rho(x)-p(x) \\
\rho(t=0, x)=\rho_{0}(x)=\int_{\mathbb{R}^{d}} f_{0}(x, v) d v
\end{array}\right.
$$

where $f_{0}$ is the weak limit of $f_{0}^{\varepsilon}$. Moreover, $(\rho, \phi)$ satisfies the free energy bound

$$
\int_{\mathbb{R}^{d}} \rho \log \rho+\frac{1}{2}|\nabla \phi|^{2}(t)+\int_{0}^{t} \int_{\mathbb{R}^{d}} \rho|\nabla(\log \rho+\phi)|^{2} \leq C .
$$

REMARK 2.2. In the case where there is no background positive charges, assumption A2 should be replaced by

$$
\int_{\mathbb{R}^{d}} \int_{\mathbb{R}^{d}} \rho_{0}^{\epsilon}(x) \rho_{0}^{\epsilon}(y)|\log | x-y|| d x d y \leq C
$$

in $2 d$ and just by the uniform boundedness of $\nabla_{x} \phi_{0}^{\varepsilon}$ in $L^{2}\left(\mathbb{R}^{d}\right)$ for $d \geq 3$.

The proof of Theorem 2.1 is as follows. In section 3 , we recall some useful properties of the Fokker-Planck operator. In section 4, we recall the existence of free energy (or renormalized) solutions to the VPFP system. Then, in section 5, we establish some a priori uniform estimates. In section 6, we prove the compactness of $\rho^{\varepsilon}$ using an averaging lemma. This result will be essential to pass to the limit in the equation which will be done in section 7. In the last section we prove the regularity estimates on $(\rho, \phi)$, which will end the proof of Theorem 2.1. 


\section{The Fokker-Planck operator}

In this section, we shall present the spectral properties of $\mathcal{L}_{F P}$ allowing us to describe the limit model. Let us define the Hilbert space $L_{M}^{2}\left(\mathbb{R}^{d}\right)$ as

$$
L_{M}^{2}\left(\mathbb{R}^{d}\right)=\left\{f \in L^{2}\left(\mathbb{R}^{d}\right) \mid \int_{\mathbb{R}^{d}} f^{2} \frac{d v}{M}<+\infty\right\},
$$

equipped with the inner product

$$
<f, g>_{L_{M}^{2}\left(\mathbb{R}^{d}\right)}=\int_{\mathbb{R}^{d}} f g \frac{d v}{M} .
$$

Let also define the space

$$
L_{M}^{2,1}\left(\mathbb{R}^{d}\right)=\left\{\left.f \in L_{M}^{2}\left(\mathbb{R}^{d}\right)\left|\int_{\mathbb{R}^{d}} M\right| \nabla_{v} \frac{f}{M}\right|^{2} d v<+\infty\right\} .
$$

The operator $\mathcal{L}_{F P}$ acting on $L_{M}^{2}\left(\mathbb{R}^{d}\right)$ is unbounded, with domain

$$
D\left(\mathcal{L}_{F P}\right)=\left\{f \in L_{M}^{2}\left(\mathbb{R}^{d}\right) \mid \nabla_{v}\left(e^{-\frac{|v|^{2}}{2}} \nabla_{v}\left(f e^{\frac{|v|^{2}}{2}}\right)\right) \in L_{M}^{2}\left(\mathbb{R}^{d}\right)\right\},
$$

and it satisfies the following.

Proposition 3.1. The operator $-\mathcal{L}_{F P}$ is self adjoint on $L_{M}^{2}\left(\mathbb{R}^{d}\right)$ and satisfies

1. $\operatorname{Ker}\left(\mathcal{L}_{F P}\right)=\mathbb{R} M$

2. $\mathcal{R}\left(\mathcal{L}_{F P}\right)=\left\{g \in L_{M}^{2}\left(\mathbb{R}^{d}\right) \mid \int_{\mathbb{R}^{d}} g(v) d v=0\right\}$,

3.For all $g \in \mathcal{R}\left(\mathcal{L}_{F P}\right)$, there exists $f \in D\left(\mathcal{L}_{F P}\right)$ such that $\mathcal{L}_{F P}(f)=g$.

This solution is unique under the solvability condition $\int_{\mathbb{R}^{d}} f(v) d v=0$. We denote it by $f=\mathcal{L}_{F P}^{-1}(g)$.

Proof. Let $f$ and $g$ be in $D\left(\mathcal{L}_{F P}\right)$. Then we have

$$
\int_{\mathbb{R}^{d}} \mathcal{L}_{F P}(f) \frac{g}{M} d v=-\int_{\mathbb{R}^{d}} M \nabla_{v}\left(\frac{f}{M}\right) \cdot \nabla_{v}\left(\frac{g}{M}\right) d v=\int_{\mathbb{R}^{d}} \mathcal{L}_{F P}(g) \frac{f}{M} d v .
$$

In particular,

$$
-\int_{\mathbb{R}^{d}} \mathcal{L}_{F P}(f) \frac{f}{M} d v=\int_{\mathbb{R}^{d}} M\left|\nabla_{v}\left(\frac{f}{M}\right)\right|^{2} d v \geq 0 .
$$

This implies that $-\mathcal{L}_{F P}$ is symmetric and positive and that $\mathcal{K} \operatorname{er}\left(\mathcal{L}_{F P}\right)=\mathbb{R} M$. Then we notice that $D\left(\mathcal{L}_{F P}\right)$ is dense in $L_{M}^{2}\left(\mathbb{R}^{d}\right)$ since $C_{0}^{\infty}\left(\mathcal{L}_{F P}\right) \subset D\left(\mathcal{L}_{F P}\right)$. Using the Riesz representation (or Lax-Milgram) theorem, we deduce that for all $f \in L_{M}^{2}\left(\mathbb{R}^{d}\right)$ there exists a unique $\psi \in L_{M}^{2,1}\left(\mathbb{R}^{d}\right)$ such that for all $\phi \in L_{M}^{2,1}\left(\mathbb{R}^{d}\right)$

$$
\int_{\mathbb{R}^{d}} M \nabla_{v}\left(\frac{\psi}{M}\right) \cdot \nabla_{v}\left(\frac{\phi}{M}\right) d v+\int_{\mathbb{R}^{d}} \frac{\psi \phi}{M} d v=\int_{\mathbb{R}^{d}} \frac{f \phi}{M} d v .
$$

Hence

$$
\left(1-\mathcal{L}_{F P}\right) \psi=f
$$


This ensures that $1-\mathcal{L}_{F P}$ is closed. Indeed, if $\left(\phi_{n},\left(1-\mathcal{L}_{F P}\right) \phi_{n}\right)$ converges to $(\phi, f)$ in $L_{M}^{2}\left(\mathbb{R}^{d}\right) \times L_{M}^{2}\left(\mathbb{R}^{d}\right)$. Then, using the Riesz representation theorem, there exists a $\psi \in L_{M}^{2,1}\left(\mathbb{R}^{d}\right)$ such that $\left(1-\mathcal{L}_{F P}\right) \psi=f$. Since $f \in L_{M}^{2}\left(\mathbb{R}^{d}\right)$ this also implies that $\psi \in$ $D\left(\mathcal{L}_{F P}\right)$. Hence, we deduce that $\left(1-\mathcal{L}_{F P}\right)\left(\phi_{n}-\psi\right)$ goes to 0 in $L_{M}^{2}\left(\mathbb{R}^{d}\right)$ which also implies that $\left(\phi_{n}-\psi\right)$ goes to 0 in $L_{M}^{2}\left(\mathbb{R}^{d}\right)$, namely $\phi=\psi \in L_{M}^{2}\left(\mathbb{R}^{d}\right)$.

Hence, we also deduce that $-\mathcal{L}_{F P}$ is closed. Moreover, -1 is in the resolvent of $-\mathcal{L}_{F P}$. Hence $-\mathcal{L}_{F P}$ is self-adjoint (see for instance [32, Chap. X, p. 137]). Using that $-\mathcal{L}_{F P}$ is self-adjoint, we deduce that $\mathcal{R}\left(\mathcal{L}_{F P}\right)=\mathcal{K} \operatorname{er}\left(\mathcal{L}_{F P}\right)^{\perp}$. Hence points 2 and 3 follow easily.

Another useful property of $\mathcal{L}_{F P}$ is taken from [30]:

Lemma 3.2. The operator $\mathcal{L}_{F P}$ satisfies, for all $f \in L^{1}(d v) \cap D\left(\mathcal{L}_{F P}\right), f \geq 0$ and $\mathcal{L}_{F P}(f), f\left(|v|^{2}+|\log (f)|\right) \in L^{1}(d v)$,

$$
\int_{\mathbb{R}^{d}} \mathcal{L}_{F P}(f) d v=0 \quad \text { and } \quad \mathcal{H}(f)=\int_{\mathbb{R}^{d}} \mathcal{L}_{F P}(f) \log \left(\frac{f}{M}\right) d v \leq 0 .
$$

Moreover,

$$
\mathcal{H}(f)=0 \Leftrightarrow \mathcal{L}_{F P}(f)=0 \Leftrightarrow f(v)=\rho M(v)
$$

\section{Existence of renormalized solutions}

Now let us give the definition of renormalized solutions.

Definition 4.1. We say that $\left(f^{\varepsilon}, \phi^{\varepsilon}\right) \in L^{\infty}\left(L^{1} \times \dot{H}^{1}\right)$ is a renormalized solution to the VPFP system (1.1)-(2.1) if it satisfies

1. $\forall \beta \in \mathcal{C}^{1}\left(\mathbb{R}^{+}\right),|\beta(t)| \leq C(\sqrt{t}+1),\left|\sqrt{t} \beta^{\prime}(t)\right| \leq C$, and $\left|t \beta^{\prime \prime}(t)\right| \leq C, \beta\left(f^{\varepsilon}\right)$ is a weak solution of

$$
\left\{\begin{array}{l}
\varepsilon \partial_{t} \beta\left(f^{\varepsilon}\right)+v \cdot \nabla_{x} \beta\left(f^{\varepsilon}\right)-\nabla_{v} \cdot\left(\nabla_{x} \phi^{\varepsilon} \beta\left(f^{\varepsilon}\right)\right)=\beta^{\prime}\left(f^{\varepsilon}\right) \frac{\mathcal{L}_{F P}\left(f^{\varepsilon}\right)}{\varepsilon} \\
\beta\left(f^{\varepsilon}\right)(t=0)=\beta\left(f_{0}^{\varepsilon}\right) . \\
-\Delta \phi^{\varepsilon}=\int f^{\varepsilon} d v-p(x) .
\end{array}\right.
$$

2. $\forall \lambda>0, \theta_{\varepsilon, \lambda}=\sqrt{f^{\varepsilon}+\lambda M}$ satisfies

$$
\varepsilon \partial_{t} \theta_{\varepsilon, \lambda}+v \cdot \nabla_{x} \theta_{\varepsilon, \lambda}-\nabla_{v} \cdot\left(\nabla_{x} \phi^{\varepsilon} \theta_{\varepsilon, \lambda}\right)=\frac{\mathcal{L}_{F P}\left(f^{\varepsilon}\right)}{2 \varepsilon \theta_{\varepsilon, \lambda}}+\frac{\lambda M}{2 \theta_{\varepsilon, \lambda}} v \cdot \nabla_{x} \phi^{\varepsilon} .
$$

Notice that we need two types of renormalization, namely $\beta\left(f^{\epsilon}\right)$ and $\gamma\left(\frac{f^{\epsilon}}{M}\right)$ where $\gamma(t)=\sqrt{t+\lambda}$. The next proposition states the existence of free energy renormalized solutions:

Proposition 4.2. The VPFP system (1.1)-(2.1) has a renormalized solution in the sense of Definition 4.1 which additionally satisfies

1. The continuity equation

$$
\partial_{t} \rho^{\varepsilon}+\nabla_{x} \cdot J^{\varepsilon}=0,
$$


2. The entropy, or more precisely the free energy inequality

$$
\begin{gathered}
\int_{\mathbb{R}^{d}} \int_{\mathbb{R}^{d}}\left(\frac{|v|^{2}}{2}+\log \left(f^{\varepsilon}\right)\right) f^{\varepsilon} d v d x+\frac{1}{2} \int_{\mathbb{R}^{d}}\left|\nabla_{x} \phi^{\varepsilon}\right|^{2} d x \\
+\frac{4}{\varepsilon^{2}} \int_{0}^{t} \int_{\mathbb{R}^{d}} \int_{\mathbb{R}^{d}}\left|\nabla_{v} \sqrt{f^{\varepsilon} e^{\frac{|v|^{2}}{2}}}\right|^{2} e^{-\frac{|v|^{2}}{2}} d v d x d s \\
\leq \int_{\mathbb{R}^{d}} \int_{\mathbb{R}^{d}}\left(\frac{|v|^{2}}{2}+\log \left(f_{0}^{\varepsilon}\right)\right) f_{0}^{\varepsilon} d v d x+\frac{1}{2} \int_{\mathbb{R}^{d}}\left|\nabla_{x} \phi_{0}^{\varepsilon}\right|^{2} d x .
\end{gathered}
$$

This solution will be called free energy (renormalized) solution.

Sketch of the proof. We will only present the formal calculation which leads to (4.4). These calculations also appear in [30]. We refer to [12, 7] for the proof of existence using those a priori estimates. The first estimate can be deduced by multiplying the first equation from VPFP system by $|v|^{2}$ and integrating the result with respect to $x$ and $v$. Thus we obtain

$$
\begin{aligned}
& \frac{d}{d t} \int_{\mathbb{R}^{d}} \int_{\mathbb{R}^{d}} \frac{|v|^{2}}{2} f^{\varepsilon} d v d x+\int_{\mathbb{R}^{d}} \int_{\mathbb{R}^{d}}\left(v \cdot \nabla_{x} \phi^{\varepsilon}\right) f^{\varepsilon} d v d x \\
= & -\frac{1}{\varepsilon^{2}} \int_{\mathbb{R}^{d}} \int_{\mathbb{R}^{d}}\left(v f^{\varepsilon}+\nabla_{v} f^{\varepsilon}\right) \cdot v d v d x
\end{aligned}
$$

the second term on the left-hand side of (4.5) can be written as follows:

$$
\begin{aligned}
\int_{\mathbb{R}^{d}} \int_{\mathbb{R}^{d}}\left(v \cdot \nabla_{x} \phi^{\varepsilon}\right) f^{\varepsilon} d v d x & =\varepsilon \int_{\mathbb{R}^{d}} \phi^{\varepsilon} \partial_{t} \rho^{\varepsilon} d x \\
& =\frac{\varepsilon}{2} \frac{d}{d t} \int_{\mathbb{R}^{d}}\left|\nabla_{x} \phi^{\varepsilon}\right|^{2} d x .
\end{aligned}
$$

Finally, the entropy inequality is formally obtained by multiplying the first equation from VPFP system by $\log \left(f^{\varepsilon}\right)$ and integrating the result with respect to $x$ and $v$. We obtain

$$
\frac{d}{d t} \int_{\mathbb{R}^{d}} \int_{\mathbb{R}^{d}} f^{\varepsilon} \log \left(f^{\varepsilon}\right) d v d x=-\frac{1}{\varepsilon^{2}} \int_{\mathbb{R}^{d}} \int_{\mathbb{R}^{d}}\left(v f^{\varepsilon}+\nabla_{v} f^{\varepsilon}\right) \cdot \frac{\nabla_{v} f^{\varepsilon}}{f^{\varepsilon}} d v d x .
$$

Therefore, summing up these relations yields

$$
\begin{aligned}
& \frac{d}{d t}\left(\int_{\mathbb{R}^{d}} \int_{\mathbb{R}^{d}}\left(\frac{|v|^{2}}{2}+\log \left(f^{\varepsilon}\right)\right) f^{\varepsilon} d v d x+\frac{1}{2} \int_{\mathbb{R}^{d}}\left|\nabla_{x} \phi^{\varepsilon}\right|^{2} d x\right) \\
= & -\frac{1}{\varepsilon^{2}} \int_{\mathbb{R}^{d}} \int_{\mathbb{R}^{d}}\left(v f^{\varepsilon}+\nabla_{v} f^{\varepsilon}\right) \cdot\left(v+\frac{\nabla_{v} f^{\varepsilon}}{f^{\varepsilon}}\right) d v d x \\
= & -\frac{1}{\varepsilon^{2}} \int_{\mathbb{R}^{d}} \int_{\mathbb{R}^{d}}\left(v \sqrt{f^{\varepsilon}}+2 \nabla_{v} \sqrt{f^{\varepsilon}}\right)^{2} d v d x \\
= & -\frac{4}{\varepsilon^{2}} \int_{\mathbb{R}^{d}} \int_{\mathbb{R}^{d}}\left|\nabla_{v} \sqrt{f^{\varepsilon} e^{\frac{|v|^{2}}{2}}}\right|^{2} e^{-\frac{|v|^{2}}{2}} d v d x .
\end{aligned}
$$

We point out that the logarithmic Sobolev inequality, implies that

$$
4 \int_{\mathbb{R}^{d}}\left|\nabla_{v} \sqrt{f^{\varepsilon} e^{\frac{|v|^{2}}{2}}}\right|^{2} e^{-\frac{|v|^{2}}{2}} d v \geq 2 \int_{\mathbb{R}^{d}} f^{\varepsilon} \log \left(\frac{f^{\varepsilon}}{\rho^{\varepsilon} M}\right) d v
$$

which yields a bound on the relative entropy of $f^{\epsilon}$ with respect to $\rho^{\epsilon} M$. 


\section{Uniform estimates}

The aim of this section is the derivation of a priori estimates, uniform with respect to $\varepsilon$, on these renormalized solutions. More precisely, we will justify the following claim.

Proposition 5.1. Assume that assumptions A1 and A2 are satisfied. Then there exists a renormalized solution $\left(f^{\varepsilon}, \phi^{\varepsilon}\right)$ of the VPFP system (1.1)-(2.1) which satisfies the conclusions of Proposition 4.2.

Additionally, the following quantities are bounded for any $t \in[0, T]$, with bounds which are independent of $\varepsilon$ and $t$ :

$$
\int_{\mathbb{R}^{d}} \int_{\mathbb{R}^{d}}\left(1+|x|+|v|^{2}+\left|\log \left(f^{\varepsilon}\right)\right|\right) f^{\varepsilon} d v d x, \int_{\mathbb{R}^{d}}\left|\nabla_{x} \phi^{\varepsilon}\right|^{2} d x
$$

and

$$
\frac{1}{\varepsilon^{2}} \int_{0}^{t} \int_{\mathbb{R}^{d}} \int_{\mathbb{R}^{d}}\left|\nabla_{v} \sqrt{f^{\varepsilon} e^{\frac{|v|^{2}}{2}}}\right|^{2} e^{-\frac{|v|^{2}}{2}} d v d x d s .
$$

Moreover, $f^{\varepsilon}$ is weakly relatively compact in $L^{1}\left((0, T) \times \mathbb{R}^{d} \times \mathbb{R}^{d}\right)$.

Proof. The proof is similar to the proof of Lemma 2.3 in [30]. From Proposition 4.2, we deduce that

$$
\mathcal{E}^{\varepsilon}(t)+\frac{4}{\varepsilon^{2}} \int_{0}^{t} \int_{\mathbb{R}^{d}} \int_{\mathbb{R}^{d}}\left|\nabla_{v} \sqrt{f^{\varepsilon} e^{\frac{|v|^{2}}{2}}}\right|^{2} e^{-\frac{|v|^{2}}{2}} d v d x d s \leq \mathcal{E}^{\varepsilon}(0) .
$$

Let

$$
\log \left(f^{\varepsilon}\right)=\log ^{+}\left(f^{\varepsilon}\right)-\log ^{-}\left(f^{\varepsilon}\right),
$$

where $\log ^{-}\left(f^{\varepsilon}\right):=\max \left\{-\log \left(f^{\varepsilon}\right), 0\right\}$ and $\log ^{+}\left(f^{\varepsilon}\right):=\max \left\{\log \left(f^{\varepsilon}\right), 0\right\}$. Then we split the domain into two parts $\left\{f^{\varepsilon}>e^{-\left(\frac{|x|}{2}+\frac{|v|^{2}}{4}\right)}\right\}$ and $\left\{f^{\varepsilon} \leq e^{-\left(\frac{|x|}{2}+\frac{|v|^{2}}{4}\right)}\right\}$. In the first part we have

$$
f^{\varepsilon} \log ^{-}\left(f^{\varepsilon}\right) \leq\left(\frac{|x|}{2}+\frac{|v|^{2}}{4}\right) f^{\varepsilon}
$$

and in the second part

$$
f^{\varepsilon} \log ^{-}\left(f^{\varepsilon}\right) \leq C \sqrt{f^{\varepsilon}} \leq C e^{-\left(\frac{|x|}{4}+\frac{|v|^{2}}{8}\right)} .
$$

Therefore we obtain

$$
\begin{gathered}
\mathcal{E}^{\varepsilon}(t)+\int_{\mathbb{R}^{d}} \int_{\mathbb{R}^{d}}|x| f^{\varepsilon} d v d x \\
\geq \int_{\mathbb{R}^{d}} \int_{\mathbb{R}^{d}}\left(\frac{|x|}{2}+\frac{|v|^{2}}{4}+\log ^{+}\left(f^{\varepsilon}\right)\right) f^{\varepsilon} d v d x-C \int_{\mathbb{R}^{d}} \int_{\mathbb{R}^{d}} e^{-\left(\frac{|x|}{2}+\frac{|v|^{2}}{4}\right)} d v d x .
\end{gathered}
$$

On the other hand, we have

$$
\begin{aligned}
\frac{d}{d t} \int_{\mathbb{R}^{d}} \int_{\mathbb{R}^{d}}|x| f^{\varepsilon} d v d x & =\frac{1}{\varepsilon} \int_{\mathbb{R}^{d}} \int_{\mathbb{R}^{d}} v \cdot \frac{x}{|x|} f^{\varepsilon} d v d x \\
& =\frac{1}{\varepsilon} \int_{\mathbb{R}^{d}} \int_{\mathbb{R}^{d}}\left(v \sqrt{f^{\varepsilon}}+2 \nabla_{v} \sqrt{f^{\varepsilon}}\right) \cdot \frac{x}{|x|} \sqrt{f^{\varepsilon}} d v d x \\
& \leq \frac{1}{2} \int_{\mathbb{R}^{d}} \int_{\mathbb{R}^{d}} f^{\varepsilon} d v d x+\frac{1}{2} \int_{\mathbb{R}^{d}} \int_{\mathbb{R}^{d}}\left|\frac{v \sqrt{f^{\varepsilon}}+2 \nabla_{v} \sqrt{f^{\varepsilon}}}{\varepsilon}\right|^{2} d v d x .
\end{aligned}
$$


Hence, we obtain

$$
\begin{gathered}
\int_{\mathbb{R}^{d}}|x| f^{\varepsilon} d v d x \leq \int_{\mathbb{R}^{d}}|x| f_{0}^{\varepsilon} d v d x \\
+\frac{t}{2}\left\|f_{0}^{\varepsilon}\right\|_{L^{1}\left(\mathbb{R}^{d} \times \mathbb{R}^{d}\right)}+\frac{2}{\varepsilon^{2}} \int_{0}^{t} \int_{\mathbb{R}^{d}} \int_{\mathbb{R}^{d}} \mid \nabla_{v} \sqrt{\left.f^{\varepsilon} e^{\frac{|v|^{2}}{2}}\right|^{2} e^{-\frac{|v|^{2}}{2}} d v d x d s .}
\end{gathered}
$$

Finally, we obtain

$$
\begin{aligned}
& \frac{1}{2} \int_{\mathbb{R}^{d}}\left|\nabla \phi^{\epsilon}\right|^{2}+\int_{\mathbb{R}^{d}} \int_{\mathbb{R}^{d}}\left(\frac{|x|}{2}+\frac{|v|^{2}}{4}+\log ^{+}\left(f^{\varepsilon}\right)\right) f^{\varepsilon} d v d x \\
& \quad+\frac{1}{2 \varepsilon^{2}} \int_{0}^{t} \int_{\mathbb{R}^{d}} \int_{\mathbb{R}^{d}}\left|\nabla_{v} \sqrt{f^{\varepsilon} e^{\frac{|v|^{2}}{2}}}\right|^{2} e^{-\frac{|v|^{2}}{2}} d v d x d s \\
& \leq C+\left\|\left(\frac{t}{2}+|x|\right) f_{0}^{\varepsilon}\right\|_{L^{1}\left(\mathbb{R}^{d} \times \mathbb{R}^{d}\right)}+\mathcal{E}^{\varepsilon}(0),
\end{aligned}
$$

which leads to the desired results.

Lemma 5.2. [30] The current density $J^{\varepsilon}$ can be estimated by

$$
\left\|J^{\varepsilon}(t, \cdot)\right\|_{L^{1}\left(\mathbb{R}^{d}\right)} \leq \frac{2}{\varepsilon^{2}} \int_{\mathbb{R}^{d}} \int_{\mathbb{R}^{d}}\left|\nabla_{v} \sqrt{f^{\varepsilon} e^{\frac{|v|^{2}}{2}}}\right|^{2} e^{-\frac{|v|^{2}}{2}} d v d x+\frac{1}{2}\left\|f_{0}^{\varepsilon}\right\|_{L^{1}\left(\mathbb{R}^{d} \times \mathbb{R}^{d}\right)} .
$$

Proof. The current density can be written as follows

$$
J^{\varepsilon}=\frac{1}{\varepsilon} \int_{\mathbb{R}^{d}}\left(v \sqrt{f^{\varepsilon}}+2 \nabla_{v} \sqrt{f^{\varepsilon}}\right) \sqrt{f^{\varepsilon}} d v
$$

and using the Cauchy-Schwartz inequality and mass conservation, we obtain the estimate.

Corollary 5.3. Assume that assumption A1 is satisfied. Then, $\left|\nabla_{v} \sqrt{f^{\varepsilon}}\right|^{2}$ is bounded in $L^{1}\left((0, T) \times \mathbb{R}^{d} \times \mathbb{R}^{d}\right)$.

Proof. We note that

$$
\begin{aligned}
0 & \leq \int_{0}^{t} \int_{\mathbb{R}^{d}} \int_{\mathbb{R}^{d}}\left|\nabla_{v} \sqrt{f^{\varepsilon}}\right|^{2} d v d x d s \\
& =\int_{0}^{t} \int_{\mathbb{R}^{d}} \int_{\mathbb{R}^{d}}\left(\frac{1}{4}\left|v \sqrt{f^{\varepsilon}}+2 \nabla_{v} \sqrt{f^{\varepsilon}}\right|^{2}-\frac{1}{4}|v|^{2} f^{\varepsilon}-v \sqrt{f^{\varepsilon}} \cdot \nabla_{v} \sqrt{f^{\varepsilon}}\right) d v d x d s \\
& \leq \frac{1}{4} \int_{0}^{t} \int_{\mathbb{R}^{d}} \int_{\mathbb{R}^{d}}\left|v \sqrt{f^{\varepsilon}}+2 \nabla_{v} \sqrt{f^{\varepsilon}}\right|^{2} d v d x d s+\frac{d}{2} \int_{0}^{t} \int_{\mathbb{R}^{d}} \int_{\mathbb{R}^{d}} f^{\varepsilon} d v d x d s .
\end{aligned}
$$

Hence, we conclude by using Proposition 5.1 .

COROLlary 5.4. The renormalized solution $\left(f^{\varepsilon}, \phi^{\varepsilon}\right)$ satisfies

1. $\rho^{\varepsilon}$ is weakly relatively compact in $L^{1}\left((0, T) \times \mathbb{R}^{d}\right)$

2. $\nabla \phi^{\varepsilon}$ is relatively compact in $L^{2}\left(0, T ; L_{l o c}^{p}\left(\mathbb{R}^{d}\right)\right)$ for all $1 \leq p<2$.

Proof. See [24] Proposition 3.3. The only difference is that here we are in the whole space in $x$ and hence we need to localize the conclusion. 
Let us define

$$
r_{\varepsilon}=\frac{1}{\varepsilon \sqrt{M}}\left(\sqrt{f^{\varepsilon}}-\sqrt{\rho^{\varepsilon} M}\right)
$$

Proposition 5.5. $\quad r_{\varepsilon}$ is such that $\left|r_{\varepsilon}\right|^{2} M$ is bounded in $L^{1}\left((0, T) \times \mathbb{R}^{d} \times \mathbb{R}^{d}\right)$, $\varepsilon\left|r_{\varepsilon}\right|^{2}|v|^{2} M$ is bounded in $L^{1}\left((0, T) \times \mathbb{R}^{d} \times \mathbb{R}^{d}\right)$, and $\sqrt{\varepsilon}\left|r_{\varepsilon}\right|^{2}|v| M$ is bounded in $L^{1}\left((0, T) \times \mathbb{R}^{d} \times \mathbb{R}^{d}\right)$.

Proof. The proof uses Young inequality (see [2] and [21] where a similar argument is used to control the distance to the Maxwellian in the hydrodynamic limit of the Boltzmann equation). Denote $h$ to be the nonnegative convex function defined for all $z \in]-1,+\infty[$ by

$$
h(z)=(1+z) \log (1+z)-z .
$$

We also denote $g_{\varepsilon}=\frac{f^{\varepsilon}}{\rho^{\varepsilon} M}$. Hence $r_{\varepsilon}=\frac{\sqrt{\rho^{\varepsilon}}}{\varepsilon}\left(\sqrt{g_{\varepsilon}}-1\right)$ is actually controlled by the relative entropy from the inequality $h(z) \geq 2(\sqrt{1+z}-1)^{2}$, which holds for all $\left.z \in\right]-$ $1,+\infty[$. We indeed deduce that

$$
r_{\varepsilon}^{2} M=\frac{\rho^{\varepsilon} M}{2 \varepsilon^{2}} 2\left(\sqrt{g_{\varepsilon}}-1\right)^{2} \leq \frac{\rho^{\varepsilon} M}{2 \varepsilon^{2}}\left(g_{\varepsilon} \log \left(g_{\varepsilon}\right)-g_{\varepsilon}+1\right) .
$$

Then we use the logarithmic Sobolev inequality, which yields

$$
\begin{aligned}
\int_{\mathbb{R}^{d}} r_{\varepsilon}^{2} M d v & \leq \frac{1}{2 \varepsilon^{2}} \int_{\mathbb{R}^{d}}\left(g_{\varepsilon} \log \left(g_{\varepsilon}\right)-g_{\varepsilon}+1\right) \rho^{\varepsilon} M d v \\
& \leq \frac{1}{2 \varepsilon^{2}} \int_{\mathbb{R}^{d}} f_{\varepsilon} \log \left(\frac{f^{\varepsilon}}{\rho^{\varepsilon} M}\right) d v \\
& \leq \frac{1}{\varepsilon^{2}} \int_{\mathbb{R}^{d}}\left|\nabla_{v} \sqrt{f^{\varepsilon} e^{\frac{|v|^{2}}{2}}}\right|^{2} e^{-\frac{|v|^{2}}{2}} d v
\end{aligned}
$$

We conclude by integrating with respect to $x$ and $t$. This proves the first assertion. Denote the Legendre transform of the convex function $h$ by $h^{*}$. An explicit computation gives $h^{*}(z)=\exp (z)-z-1$ for all $z \in \mathbb{R}$. In particular, $h^{*}$ is superquadratic: $h^{*}(\lambda z) \leq \lambda^{2} h^{*}(z)$ for all $\lambda \in[0,1]$ and all $z \geq 0$. By Young's inequality,

$$
\begin{aligned}
\varepsilon\left|r_{\varepsilon}\right|^{2}|v|^{2} & \leq \frac{\rho^{\varepsilon}}{\varepsilon}\left|g_{\varepsilon}-1\right||v|^{2} \\
& \leq \frac{4 \rho^{\varepsilon}}{\varepsilon^{2}}\left[h\left(\left|g_{\varepsilon}-1\right|\right)+h^{*}\left(\frac{\varepsilon}{4}|v|^{2}\right)\right] \\
& \leq \frac{4 \rho^{\varepsilon}}{\varepsilon^{2}}\left[h\left(g_{\varepsilon}-1\right)+\varepsilon^{2} h^{*}\left(\frac{|v|^{2}}{4}\right)\right],
\end{aligned}
$$

from which we deduce that

$$
\begin{aligned}
\varepsilon \int_{\mathbb{R}^{d}}\left|r_{\varepsilon}\right|^{2}|v|^{2} M d v \leq \frac{8}{\varepsilon^{2}} \int_{\mathbb{R}^{d}}\left|\nabla_{v} \sqrt{f^{\varepsilon} e^{\frac{|v|^{2}}{2}}}\right|^{2} e^{-\frac{|v|^{2}}{2}} d v \\
+4 \rho^{\varepsilon} \int_{\mathbb{R}^{d}} \exp \left(-\frac{|v|^{2}}{4}\right) d v .
\end{aligned}
$$

The third assertion is a consequence of the Cauchy-Schwarz inequality which gives

$$
\sqrt{\varepsilon}\left|r_{\varepsilon}\right|^{2}|v| M \leq \frac{1}{2}\left|r_{\varepsilon}\right|^{2} M+\frac{\varepsilon}{2}\left|r_{\varepsilon}\right|^{2}|v|^{2} M
$$


We conclude by using the first and second bound. This ends the proof of the proposition.

6. Compactness of the density

Proposition 6.1. The density $\rho^{\varepsilon}$ is relatively compact in $L^{1}\left((0, T) \times \mathbb{R}^{d}\right)$. There exists $\rho \in L^{1}\left((0, T) \times \mathbb{R}^{d}\right)$ such that, up to extraction of a subsequence if necessary,

$$
\rho^{\varepsilon} \rightarrow \rho \text { in } L^{1} \text { and a.e. }
$$

In the following, we will use

$$
\beta(s)=\frac{s}{1+s}, \quad \beta_{\delta}(s)=\frac{1}{\delta} \beta(\delta s), \quad \forall s>0 .
$$

We recall that for every fixed $\delta>0$, we have

1. $0 \leq \beta_{\delta}(s) \leq \min \left(s, \frac{1}{\delta}\right)$,

2. $\quad\left|\beta_{\delta}(s)\right| \leq C_{\delta}(\sqrt{s}+1)$,

3. $\left|\sqrt{s} \beta_{\delta}^{\prime}(s)\right| \leq C_{\delta}$,

4. $\quad\left|s \beta_{\delta}^{\prime \prime}(s)\right| \leq C_{\delta}$.

We remark that if we want to prove Proposition 6.1, we only need to show for all $\delta>0$, the compactness of the density associated to $\left(\beta_{\delta}\left(f^{\varepsilon}\right)\right)_{\varepsilon}$. This is a consequence of the following averaging lemma (see [16, 14]).

Lemma 6.2. Assume that $h^{\varepsilon}$ is bounded in $L^{2}\left((0, T) \times \mathbb{R}^{d} \times \mathbb{R}^{d}\right), h_{0}^{\varepsilon}$ and $h_{1}^{\varepsilon}$ are bounded in $L^{1}\left((0, T) \times \mathbb{R}^{d} \times \mathbb{R}^{d}\right)$, and

$$
\varepsilon \partial_{t} h^{\varepsilon}+v \cdot \nabla_{x} h^{\varepsilon}=h_{0}^{\varepsilon}+\nabla_{v} \cdot h_{1}^{\varepsilon} .
$$

Then, for all $\psi \in \mathcal{C}_{0}^{\infty}\left(\mathbb{R}^{d}\right)$,

$$
\left\|\int_{\mathbb{R}^{d}}\left(h^{\varepsilon}(t, x+y, v)-h^{\varepsilon}(t, x, v)\right) \psi(v) d v\right\|_{L_{t, x}^{1}} \rightarrow 0
$$

when $y \rightarrow 0$ uniformly in $\varepsilon$.

We refer to [24] for the proof of this lemma.

\section{Proof of Proposition 6.1.}

Proof. Let $\delta$ be a (fixed) nonnegative parameter. Let us verify that the rescaled VPFP system (in the renormalized sense) satisfies the assumptions of the previous lemma. Indeed, $\beta_{\delta}\left(f^{\varepsilon}\right)$ is a weak solution of

$$
\varepsilon \partial_{t} \beta_{\delta}\left(f^{\varepsilon}\right)+v \cdot \nabla_{x} \beta_{\delta}\left(f^{\varepsilon}\right)=h_{0}^{\varepsilon}+\nabla_{v} \cdot h_{1}^{\varepsilon},
$$

where

$$
h_{0}^{\varepsilon}=-\frac{\left(v f^{\varepsilon}+\nabla_{v} f^{\varepsilon}\right)}{\varepsilon} \nabla_{v} f^{\varepsilon} \beta_{\delta}^{\prime \prime}\left(f^{\varepsilon}\right)
$$

and

$$
h_{1}^{\varepsilon}=\nabla_{x} \phi^{\varepsilon} \beta_{\delta}\left(f^{\varepsilon}\right)+\frac{\left(v f^{\varepsilon}+\nabla_{v} f^{\varepsilon}\right)}{\varepsilon} \beta_{\delta}^{\prime}\left(f^{\varepsilon}\right) .
$$


The sequence $\left(\beta_{\delta}\left(f^{\varepsilon}\right)\right)_{\varepsilon}$ is bounded in $L^{\infty} \cap L^{1}\left((0, T) \times \mathbb{R}^{d} \times \mathbb{R}^{d}\right)$ and hence in $L^{2}\left((0, T) \times \mathbb{R}^{d} \times \mathbb{R}^{d}\right)$. Moreover, by applying Hölder's inequality and using the uniform bound of $\beta_{\delta}\left(f^{\varepsilon}\right)$ in $L^{2}$ (for fixed $\delta$ ) and by using the uniform estimates: $\frac{\left(v f^{\varepsilon}+\nabla_{v} f^{\varepsilon}\right)}{\varepsilon \sqrt{f^{\varepsilon}}}$, $\nabla_{v} \sqrt{f^{\varepsilon}}$, and $\nabla_{x} \phi^{\varepsilon}$ are bounded in $L^{2}$, we see that

$$
h^{\varepsilon}:=\beta_{\delta}\left(f^{\varepsilon}\right), h_{0}^{\varepsilon}=-2 \frac{\left(v f^{\varepsilon}+\nabla_{v} f^{\varepsilon}\right)}{\varepsilon \sqrt{f^{\varepsilon}}} \nabla_{v} \sqrt{f^{\varepsilon}} \beta_{\delta}^{\prime \prime}\left(f^{\varepsilon}\right) f^{\varepsilon}
$$

and

$$
h_{1}^{\varepsilon}=\nabla_{x} \phi^{\varepsilon} \beta_{\delta}\left(f^{\varepsilon}\right)+\frac{\left(v f^{\varepsilon}+\nabla_{v} f^{\varepsilon}\right)}{\varepsilon \sqrt{f^{\varepsilon}}} \beta_{\delta}^{\prime}\left(f^{\varepsilon}\right) \sqrt{f^{\varepsilon}}
$$

satisfy the assumptions of the above lemma. Applying this lemma we deduce the compactness in $x$ of $\int_{\mathbb{R}^{d}} \beta_{\delta}\left(f^{\varepsilon}\right) \psi(v) d v$ for all $\psi \in \mathcal{D}\left(\mathbb{R}^{d}\right)$, namely (6.2) holds with $h^{\varepsilon}$ replaced by $\beta_{\delta}\left(f^{\varepsilon}\right)$.

Next, using that $\left(\beta_{\delta}\left(f^{\varepsilon}\right)\right)_{\varepsilon}$ is bounded in $L^{\infty}\left(0, T ; L^{1}\left(\left(1+|v|^{2}\right) d x d v\right)\right)$, we see that we can take $\psi(v)$ to be a constant equal to 1 in (6.2) and hence we deduce, after also sending $\delta$ to 0 and using the equi-integrability of $f^{\varepsilon}$, that

$$
\left\|\rho^{\varepsilon}(t, x+y)-\rho^{\varepsilon}(t, x)\right\|_{L_{t, x}^{1}} \rightarrow 0 \text { when } y \rightarrow 0 \text { uniformlyin } \varepsilon .
$$

Finally, using that $\partial_{t} \rho^{\varepsilon}=-\nabla_{x} \cdot J^{\varepsilon}$ is bounded in $L^{1}\left(0, T ; W^{-1,1}\left(\mathbb{R}^{d}\right)\right)$, we deduce that $\rho^{\varepsilon}$ is relatively compact in $L^{1}\left((0, T) \times \mathbb{R}^{d}\right)$ which ends the proof of the proposition. We also point out here that the bound $\partial_{t} \rho^{\varepsilon}$ in $L^{1}\left(0, T ; W^{-1,1}\left(\mathbb{R}^{d}\right)\right)$ justifies that we can also pass to the limit in the initial data, and that $\rho(t=0)=\rho_{0}$.

\section{Passage to the limit}

We would like to pass to the limit in the continuity equation

$$
\partial_{t} \rho^{\varepsilon}+\nabla_{x} \cdot J^{\varepsilon}=0
$$

The question is to identify the limit of the current density. Let us denote $J$ to be the weak limit of $J^{\varepsilon}$ when $\varepsilon$ goes to zero and $r$ is the weak limit of $r_{\varepsilon}$ in $L^{2}\left((0, T) \times \mathbb{R}^{d} \times\right.$ $\left.\mathbb{R}^{d}, M(v) d t d x d v\right)$, then we have the following proposition

Proposition 7.1. The electric current satisfies

$$
J^{\varepsilon}-2 \sqrt{\rho} \int_{\mathbb{R}^{d}} r v M d v, \text { in } L_{t, x}^{1}
$$

Proof. Using the previous section, there exists $\rho \in L^{1}\left((0, T) \times \mathbb{R}^{d}\right)$ such that

$$
\rho^{\varepsilon} \rightarrow \rho \text { in } L_{t, x}^{1} \text { anda.e. }
$$

The inequality $(\sqrt{a}-\sqrt{b})^{2} \leq|a-b|$ leads to

$$
\sqrt{\rho^{\varepsilon}} \rightarrow \sqrt{\rho} \text { in } L_{t, x}^{2} \text { and a.e. }
$$

The entropy dissipation given by (4.4) leads to

$$
f^{\varepsilon} \rightarrow \rho M \text { in } L_{t, x, v}^{1} \text { anda.e. }
$$


Indeed, we expand

$$
f^{\varepsilon}-\rho M=\left(f^{\varepsilon}-\rho^{\varepsilon} M\right)+\left(\rho^{\varepsilon}-\rho\right) M
$$

where we already know that $\rho^{\varepsilon}-\rho$ tends to 0 , in $L_{t, x}^{1}$. Hence, it remains to study the difference $f^{\varepsilon}-\rho^{\varepsilon} M$. This can be controlled by the entropy dissipation. We use the logarithmic Sobolev inequality as in [17], which yields

$$
\int_{\mathbb{R}^{d}} \int_{\mathbb{R}^{d}} f^{\varepsilon} \log \left(\frac{f^{\varepsilon}}{\rho^{\varepsilon} M}\right) d v d x \leq 2 \int_{\mathbb{R}^{d}} \int_{\mathbb{R}^{d}}\left|\nabla_{v} \sqrt{f^{\varepsilon} e^{\frac{|v|^{2}}{2}}}\right|^{2} e^{-\frac{|v|^{2}}{2}} d v d x,
$$

and we conclude by using the Csiszar-Kullback-Pinsker inequality (see [10, 20, 17]), which implies that

$$
\left(\int_{\mathbb{R}^{d}} \int_{\mathbb{R}^{d}}\left|f^{\varepsilon}-\rho^{\varepsilon} M\right| d v d x\right)^{2} \leq 4 \int_{\mathbb{R}^{d}} \int_{\mathbb{R}^{d}} f^{\varepsilon} \log \left(\frac{f^{\varepsilon}}{\rho^{\varepsilon} M}\right) d v d x .
$$

Using $r_{\varepsilon}$, we can write

$$
f^{\varepsilon}=\rho^{\varepsilon} M+2 \varepsilon M \sqrt{\rho^{\varepsilon}} r_{\varepsilon}+\varepsilon^{2} r_{\varepsilon}^{2} M
$$

Then, we obtain

$$
J^{\varepsilon}=2 \sqrt{\rho^{\varepsilon}} \int_{\mathbb{R}^{d}} r_{\varepsilon} v M d v+\varepsilon \int_{\mathbb{R}^{d}} r_{\varepsilon}^{2} v M d v .
$$

Thus, using Proposition 5.5 leads to

$$
J^{\varepsilon}=2 \sqrt{\rho^{\varepsilon}} \int_{\mathbb{R}^{d}} r_{\varepsilon} v M d v+\mathcal{O}(\sqrt{\varepsilon})_{L_{t, x, v}^{1}} \rightarrow 2 \sqrt{\rho} \int_{\mathbb{R}^{d}} r v M d v \text { in } L_{t, x}^{1} .
$$

We denote $\chi$ to be the unique solution in $\left[\mathcal{R}\left(\mathcal{L}_{F P}\right) \cap D\left(\mathcal{L}_{F P}\right)\right]^{d}$ of

$$
\mathcal{L}_{F P} \chi=v M .
$$

It is well known that $\chi_{i}=-v_{i} M$.

Proposition 7.2.

$$
J^{\varepsilon} \rightarrow_{D^{\prime}} J:=-2 \sqrt{\rho} \boldsymbol{D} \cdot\left(\nabla_{x} \sqrt{\rho}+\frac{1}{2} \nabla_{x} \phi \sqrt{\rho}\right),
$$

where $\boldsymbol{D}$ is the diffusion matrix defined by $\boldsymbol{D}=-\int_{\mathbb{R}^{d}} \chi(v) \otimes v d v=\mathbb{I}$.

Proof. Using that $\rho^{\varepsilon}$ converges to $\rho$ up to extraction of a subsequence, we deduce that $\theta_{\varepsilon, \lambda}$ converges to $\sqrt{(\rho+\lambda) M}$. Then using that $\frac{\mathcal{L}_{F P}\left(f^{\varepsilon}\right)}{2 \varepsilon}=\mathcal{L}_{F P}\left(M \sqrt{\rho} r_{\epsilon}+\frac{1}{2} r_{\epsilon}^{2} M\right)$, we deduce that it converges to $\sqrt{\rho} \mathcal{L}_{F P}(r M)$. Hence, we can pass to the limit in (4.2) for $\lambda>0$, up to extraction of a subsequence, and we obtain

$$
v \cdot \nabla_{x} \sqrt{(\rho+\lambda) M}-\nabla_{v} \cdot\left(\nabla_{x} \phi \sqrt{(\rho+\lambda) M}\right)=\frac{\sqrt{\rho} \mathcal{L}_{F P}(r M)}{\sqrt{(\rho+\lambda) M}}+\frac{\lambda M v \cdot \nabla_{x} \phi}{2 \sqrt{(\rho+\lambda) M}},
$$

where $\nabla_{x} \phi$ is the $L_{t, x}^{2}$-weak limit of $\nabla_{x} \phi^{\varepsilon}$. Sending $\lambda$ to 0 , we infer that

$$
\left(\nabla_{x} \sqrt{\rho}+\frac{1}{2} \nabla_{x} \phi \sqrt{\rho}\right) \cdot v M=\mathcal{L}_{F P}(r M) .
$$


Let us go back to the expression of the current density computed in Proposition 7.1. Using (7.2), we obtain

$$
\begin{aligned}
J & =2 \sqrt{\rho} \int_{\mathbb{R}^{d}} r v M d v \\
& =2 \sqrt{\rho} \int_{\mathbb{R}^{d}} r M \frac{\mathcal{L}_{F P} \chi}{M} d v \\
& =2 \sqrt{\rho} \int_{\mathbb{R}^{d}} \mathcal{L}_{F P}(r M) \chi \frac{d v}{M} \\
& =2 \sqrt{\rho} \int_{\mathbb{R}^{d}}\left[\left(\nabla_{x} \sqrt{\rho}+\frac{1}{2} \nabla_{x} \phi \sqrt{\rho}\right) \cdot v M\right] \chi \frac{d v}{M} \\
& =-2 \sqrt{\rho}\left[-\int_{\mathbb{R}^{d}} \chi \otimes v d v\right] \cdot\left(\nabla_{x} \sqrt{\rho}+\frac{1}{2} \nabla_{x} \phi \sqrt{\rho}\right) \\
& =-2 \sqrt{\rho} \cdot\left(\nabla_{x} \sqrt{\rho}+\frac{1}{2} \nabla_{x} \phi \sqrt{\rho}\right) .
\end{aligned}
$$

Now we would like to explain how we can rewrite the current $J$. More precisely, we shall prove that the limit $\rho \in L^{2}\left(0, T ; L^{2}\left(\mathbb{R}^{d}\right)\right)$ and that $\sqrt{\rho} \in L^{2}\left(0, T ; H^{1}\left(\mathbb{R}^{d}\right)\right)$.

Lemma 7.3. Let $\rho$ be a positive function such that $\rho \in L^{\infty}\left(0, T ; L^{1}\left(\mathbb{R}^{d}\right)\right)$, satisfying

$$
\left\{\begin{array}{l}
\nabla_{x} \sqrt{\rho}+\frac{1}{2} \nabla_{x} \phi \sqrt{\rho}=G \in L^{2}\left(0, T ; L^{2}\left(\mathbb{R}^{d}\right)\right), \\
-\triangle_{x} \phi=\rho \\
\nabla_{x} \phi \in L^{\infty}\left(0, T ; L^{2}\left(\mathbb{R}^{d}\right)\right) .
\end{array}\right.
$$

Then

$$
\rho \in L^{2}\left(0, T ; L^{2}\left(\mathbb{R}^{d}\right)\right), \quad \sqrt{\rho} \in L^{2}\left(0, T ; H^{1}\left(\mathbb{R}^{d}\right)\right),
$$

and

$$
\nabla_{x} \phi \sqrt{\rho} \in L^{2}\left(0, T ; L^{2}\left(\mathbb{R}^{d}\right)\right)
$$

Proof. We refer to [24] for the proof of this lemma.

Now, using the previous lemma, we can see easily that we can rewrite the current

$$
\begin{aligned}
J & =-2 \sqrt{\rho}\left[\nabla_{x} \sqrt{\rho}+\frac{1}{2} \sqrt{\rho} \nabla_{x} \phi\right] \\
& =-\left[\nabla_{x} \rho+\rho \nabla_{x} \phi\right] .
\end{aligned}
$$

This ends the proof of the main Theorem 2.1.

Acknowledgment. The authors would like to thank the two referees for many constructive remarks.

\section{REFERENCES}

[1] A. Arnold, J.A. Carrillo, I. Gamba and C.W. Shu, Low and high field scaling limits for the Vlasov- and Wigner-Poisson-Fokker-Planck systems, Transport Theory Statist. Phys., 30(2-3), 121-153, 2001. The Sixteenth International Conference on Transport Theory, Part I (Atlanta, GA, 1999). 
[2] C. Bardos, F. Golse and C.D. Levermore, Fluid dynamic limits of kinetic equations. II. Convergence proofs for the Boltzmann equation, Commun. Pure Appl. Math., 46(5), 667-753, 1993.

[3] L.L. Bonilla, J.A. Carrillo and J. Soler, $H$-theorem for electrostatic or self-gravitating VlasovPoisson-Fokker-Planck systems, Phys. Lett. A, 212(1-2), 55-59, 1996.

[4] L.L. Bonilla and J.S. Soler, High-field limit of the Vlasov-Poisson-Fokker-Planck system: a comparison of differential perturbation methods, Math. Models Methods Appl. Sci., 11(8), 1457-1468, 2001.

[5] F. Bouchut, Existence and uniqueness of a global smooth solution for the Vlasov-PoissonFokker-Planck system in three dimensions, J. Funct. Anal., 111(1), 239-258, 1993.

[6] F. Bouchut, Smoothing effect for the non-linear Vlasov-Poisson-Fokker-Planck system, J. Differ. Equs., 122(2), 225-238, 1995.

[7] F. Bouchut and J. Dolbeault, On long time asymptotics of the Vlasov-Fokker-Planck equation and of the Vlasov-Poisson-Fokker-Planck system with Coulombic and Newtonian potentials, Differential Integral Equations, 8(3), 487-514, 1995.

[8] J.A. Carrillo and J. Soler, On the initial value problem for the Vlasov-Poisson-Fokker-Planck system with initial data in $L^{p}$ spaces, Math. Methods Appl. Sci., 18(10), 825-839, 1995.

[9] J.A. Carrillo, J. Soler and J.L. Vázquez, Asymptotic behaviour for the frictionless VlasovPoisson-Fokker-Planck system, C.R. Acad. Sci. Paris Sér. I Math., 321(9), 1195-1200, 1995.

[10] I. Csiszár, Information-type measures of difference of probability distributions and indirect observations, Studia Sci. Math. Hungar., 2, 299-318, 1967.

[11] P. Degond, Global existence of smooth solutions for the Vlasov-Fokker-Planck equation in 1 and 2 space dimensions, Ann. Sci. École Norm. Sup. (4), 19(4), 519-542, 1986.

[12] R. DiPerna and P.L. Lions, Solutions globales d'équations du type Vlasov-Poisson, C.R. Acad. Sci. Paris Sér. I Math., 307(12), 655-658, 1988.

[13] R.J. DiPerna and P.L. Lions, On the Fokker-Planck-Boltzmann equation, Commun. Math. Phys., 120(1), 1-23, 1988.

[14] R.J. DiPerna, P.L. Lions and Y. Meyer, $L^{p}$ regularity of velocity averages, Ann. Inst. H. Poincaré Anal. Non Linéaire, 8(3-4), 271-287, 1991.

[15] J. Dolbeault, Free energy and solutions of the Vlasov-Poisson-Fokker-Planck system: external potential and confinement (large time behavior and steady states), J. Math. Pures Appl., (9), 78(2), 121-157, 1999.

[16] F. Golse, P.L. Lions, B. Perthame and R. Sentis, Regularity of the moments of the solution of a transport equation, J. Funct. Anal., 76(1), 110-125, 1988.

[17] T. Goudon, Hydrodynamic limit for the Vlasov-Poisson-Fokker-Planck system: analysis of the two-dimensional case, Math. Models Methods Appl. Sci., 15(5), 737-752, 2005.

[18] T. Goudon, J. Nieto, F. Poupaud and J. Soler, Multidimensional high-field limit of the electrostatic Vlasov-Poisson-Fokker-Planck system, J. Differ. Equs., 213(2), 418-442, 2005.

[19] E. Horst, On the asymptotic growth of the solutions of the Vlasov-Poisson system, Math. Methods Appl. Sci., 16(2), 75-86, 1993.

[20] S. Kullback, Information theory and statistics, Dover Publications Inc., Mineola, NY, 1997. Reprint of the second (1968) edition.

[21] P.L. Lions and N. Masmoudi, From the Boltzmann equations to the equations of incompressible fluid mechanics. I, II, Arch. Ration. Mech. Anal., 158(3), 173-193, 195-211, 2001.

[22] P.L. Lions and B. Perthame, Propagation of moments and regularity for the 3-dimensional Vlasov-Poisson system, Invent. Math., 105(2), 415-430, 1991.

[23] A.J. Majda, G. Majda and Y.X. Zheng, Concentrations in the one-dimensional Vlasov-Poisson equations I. Temporal development and non-unique weak solutions in the single component case, Phys. D, 74(3-4), 268-300, 1994.

[24] N. Masmoudi and M.L. Tayeb, Diffusion limit of a semiconductor Boltzmann-Poisson system, SIAM J. Math. Anal., 38(6), 1788-1807 (electronic), 2007.

[25] H. Neunzert, M. Pulvirenti and L. Triolo, On the Vlasov-Fokker-Planck equation, Math. Methods Appl. Sci., 6(4), 527-538, 1984.

[26] J. Nieto, F. Poupaud and J. Soler, High-field limit for the Vlasov-Poisson-Fokker-Planck system, Arch. Ration. Mech. Anal., 158(1), 29-59, 2001.

[27] K. Ono and W.A. Strauss, Regular solutions of the Vlasov-Poisson-Fokker-Planck system, Discrete Contin. Dynam. Systems, 6(4), 751-772, 2000.

[28] K. Pfaffelmoser, Global classical solutions of the Vlasov-Poisson system in three dimensions for general initial data, J. Differ. Equs., 95(2), 281-303, 1992.

[29] F. Poupaud, Diffusion approximation of the linear semiconductor Boltzmann equation: analysis of boundary layers, Asymptotic Anal., 4(4), 293-317, 1991. 
[30] F. Poupaud and J. Soler, Parabolic limit and stability of the Vlasov-Fokker-Planck system, Math. Models Methods Appl. Sci., 10(7), 1027-1045, 2000.

[31] M. Pulvirenti and C. Simeoni, $L^{\infty}$-estimates for the Vlasov-Poisson-Fokker-Planck equation, Math. Methods Appl. Sci., 23(10), 923-935, 2000.

[32] M. Reed and B. Simon, Methods of Modern Mathematical Physics. II. Fourier Analysis, SelfAdjointness, Academic Press [Harcourt Brace Jovanovich Publishers], New York, 1975.

[33] G. Rein and J. Weckler, Generic global classical solutions of the Vlasov-Fokker-Planck-Poisson system in three dimensions, J. Differ. Equs., 99(1), 59-77, 1992.

[34] J. Schaeffer, Global existence of smooth solutions to the Vlasov-Poisson system in three dimensions, Commun. Part. Differ. Equs., 16(8-9), 1313-1335, 1991.

[35] H.D. Victory, Jr. On the existence of global weak solutions for Vlasov-Poisson-Fokker-Planck systems, J. Math. Anal. Appl., 160(2), 525-555, 1991.

[36] H.D. Victory, Jr. and B.P. O'Dwyer, On classical solutions of Vlasov-Poisson Fokker-Planck systems, Indiana Univ. Math. J., 39(1), 105-156, 1990.

[37] Y.X. Zheng and A. Majda, Existence of global weak solutions to one-component Vlasov-Poisson and Fokker-Planck-Poisson systems in one space dimension with measures as initial data, Commun. Pure Appl. Math., 47(10), 1365-1401, 1994. 